\title{
ADP-ribosyltransferase PARP11 suppresses Zika virus in synergy with PARP12
}

\author{
Lili Li ${ }^{1,2}$, Yueyue Shi ${ }^{1,2}$, Sirui Li $i^{3}$, Junxiao Liu², Shulong $\mathrm{Zu}^{1,2}$, Xin Xu ${ }^{1,2}$, Meiling Gao ${ }^{1,2}$, Nina Sun ${ }^{4}$, Chaohu Pan ${ }^{1,2}$, \\ Linan Peng ${ }^{2}$, Heng Yang ${ }^{1,2^{*}}$ (D) and Genhong Cheng ${ }^{5^{*}}$
}

\begin{abstract}
Background: Zika virus (ZIKV) infection and ZIKV epidemic have been continuously spreading silently throughout the world and its associated microcephaly and other serious congenital neurological complications poses a significant global threat to public health. Type I interferon response to ZIKV infection in host cells suppresses viral replication by inducing the expression of interferon-stimulated genes (ISGs).
\end{abstract}

Methods: The study aims to demonstrate the anti-ZIKV mechanism of PARP11. PARP11 knock out and overex-

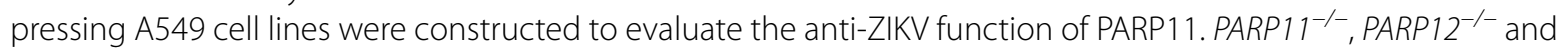
PARP11 $1^{-/-}$PARP $12^{-/-}$HEK293T cell lines were constructed to explain the synergistic effect of PARP11 and PARP12 on NS1 and NS3 protein degradation. Western blotting, immunofluorescence and immunoprecipitation assay were performed to illustrate the interaction between PARP11 and PARP12.

Results: Both mRNA and protein levels of PARP11 were induced in WT but not IFNAR1 ${ }^{-1}$ cells in response to IFNa or IFN $\beta$ stimulation and ZIKV infection. ZIKV replication was suppressed in cells expressed PARP11 but was enhanced in PARP $11^{-/-}$cells. PARP11 suppressed ZIKV independently on itself PARP enzyme activity. PARP11 interacted with PARP12 and promoted PARP12-mediated ZIKV NS1 and NS3 protein degradation.

Conclusion: We identified ADP-ribosyltransferase PARP11 as an anti-ZIKV ISG and found that it cooperated with PARP12 to enhance ZIKV NS1 and NS3 protein degradation. Our findings have broadened the understanding of the anti-viral function of ADP-ribosyltransferase family members, and provided potential therapeutic targets against viral ZIKV infection.

Keywords: ADP-ribosyltransferase, PARP11, PARP12, Zika virus, NS1 and NS3, Anti-viral ISGs

\section{Introduction}

Zika virus (ZIKV) was first isolated in 1947 in the Zika forest of Uganda from an infected rhesus macaque [1]. Since its discovery, ZIKV stayed relatively silent for almost 70 years until recent outbreaks in Pacific Islands

\footnotetext{
*Correspondence: yhmyt@hotmail.com; gcheng@mednet.ucla.edu ${ }^{1}$ Center for Systems Medicine, Institute of Basic Medical Sciences, Chinese Academy of Medical Sciences and Peking Union Medical College, Beijing 100005, China

${ }^{5}$ Department of Microbiology, Immunology and Molecular Genetics, University of California, Los Angeles, CA 90095, USA

Full list of author information is available at the end of the article
}

and Brazil. By December 2015, 18 states of Brazil had reported autochthonous ZIKV transmission and large numbers of cases of infection and its associated diseases such as microcephaly were reported in 2015 and 2016 [2-4]. On February 1, 2016, the World Health Organization (WHO) declared ZIKV outbreak and its associated clinical manifestations as a Public Health Emergency of International Concern (PHEIC). ZIKV continues to develop and spread silently throughout the world in the form of asymptomatic infections. During SeptemberNovember 2018, the biggest Indian break was reported from Rajasthan and Madhya Pradesh states of India. Up to July 2019, 87 countries reported ZIKV transmission

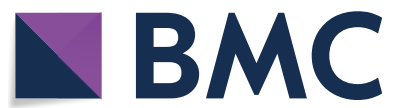

(c) The Author(s) 2021. This article is licensed under a Creative Commons Attribution 4.0 International License, which permits use, sharing, adaptation, distribution and reproduction in any medium or format, as long as you give appropriate credit to the original author(s) and the source, provide a link to the Creative Commons licence, and indicate if changes were made. The images or other third party material in this article are included in the article's Creative Commons licence, unless indicated otherwise in a credit line to the material. If material is not included in the article's Creative Commons licence and your intended use is not permitted by statutory regulation or exceeds the permitted use, you will need to obtain permission directly from the copyright holder. To view a copy of this licence, visit http://creativeco mmons.org/licenses/by/4.0/. The Creative Commons Public Domain Dedication waiver (http://creativecommons.org/publicdomain/ zero/1.0/) applies to the data made available in this article, unless otherwise stated in a credit line to the data. 
including 1,274,974 diagnosed cases in Brazil [5]. Recently, an Africa strain ZIKV infection was found in Brazil in addition to the prevalent Asia strain, suggesting more attention should be paid to another outbreak of ZIKV epidemic [6].

ZIKV infection is asymptomatic in up to $80 \%$ adults, the remaining $20 \%$ infected adults are characterized by low fever, arthralgia, maculopapular rash accompanied by pruritis, and conjunctivitis. Moreover, ZIKV infection in adults was associated with Guillain-Barre syndrome and infection during pregnancy can cause infants' microcephaly, intrauterine growth restriction and other birth defects [7-9]. Thousands of increased cases of fetal abnormalities, including microcephaly, were reported up to February 2016 in ZIKV infected areas [9-11]. At present, vaccines or antivirals to treat ZIKV infection are unavailable [12-14]. Thereby, a comprehensive research for anti-ZIKV genes and more potential therapy targets are urgently needed to be identified.

Type I interferon (IFN-I) and interferon-stimulated genes (ISGs) constitute the vital part of innate immune system against virus infection in vertebrate. Viral infection induces the production of IFN-I and about 300 ISGs which exert a broad-spectrum anti-viral effect. The potential of IFN-I and ISGs against ZIKV infection has been indicated [15-17]. Our previously work also identified $\mathrm{CH} 25 \mathrm{H}$ and PARP12 as critical anti-ZIKV ISGs, which suppressed ZIKV infection and replication [18, 19]. However, additional ISGs are likely involved in controlling ZIKV replication. The family of poly-adenosine 5'-diphosphate (ADP)-ribose polymerases (PARPs), also known as ADP-ribosyltransferases, mediates a unique translational modification called ADP-ribosylation by transferring of ADP-ribose from nicotinamide adenine dinucleotide $\left(\mathrm{NAD}^{+}\right)$to target proteins [20-22]. Among the 17 PARPs expressed in human cells, several PARPs, such as PARP13, PARP9, PARP10, PARP14, PARP12 and PARP5, have been identified as ISGs and are involved in anti-viral response [23-25]. In an anti-ZIKV ISG screening, we reported mono ADP-ribosyltransferase PARP11 with anti-ZIKV function but without detailed mechanism described [18]. On the other hand, Guo et al. reported that PARP11 promotes vesicular stomatitis virus (VSV) and herpes simplex virus-1 (HSV-1) infection by inhibiting the interferon response [26]. These results indicate a complex involvement of PARP11 in different viral infection.

In this work, we found that PARP11 was up-regulated in response to IFN-I stimulation and ZIKV infection, and acted as an anti-ZIKV ISG. Unlike PARP12, which suppressed ZIKV replication through PARP enzymatic activity dependent degradation of ZIKV NS1 and NS3 proteins, PARP11 suppressed ZIKV replication independent on its PARP enzymatic activity. Instead, PARP11 interacted and cooperated with PARP12 in suppressing ZIKV replication, which provided a new insight on understanding the mechanisms responsible for different PARP family members in viral infection.

\section{Results}

The expression of PARP11 can be induced by IFN and ZIKV infection

In our previously screening for ISGs with activity against ZIKV, we have identified PARP12 that suppresses ZIKV through PARP-dependent degradation of NS1 and NS3 viral proteins [18]. We also noticed that PARP11 showed anti-ZIKV activity. PARP11 was reported as an ISG [26]. To characterize the induction of PARP11 by IFN-I, we stimulated WT and IFN $\alpha / \beta$ receptor subunit 1 (IFNAR1)-deficient HEK293T and A549 cells with human IFN-I (IFN- $\alpha$ and IFN- $\beta$ ) and quantified the mRNA and protein levels of PARP11 by quantitative real-time PCR (qRT-PCR) and western blotting assay. We found that the PARP11 mRNA and protein levels were induced by IFN-I in WT HEK293T and A549 cells but not in the corresponding IFNAR1 $1^{-/-}$cells (Fig. 1af). Similar to exogenous IFN treatment, ZIKV infection also induced PARP11 mRNA and protein expressions in WT but not in IFNAR1 ${ }^{-/-}$A549 cells (Fig. 1g-i). These results indicate that PARP11 is an ISG induced by IFN-I and ZIKV infection.

\section{PARP11 suppresses ZIKV replication in vitro}

To further identify the anti-ZIKV activity, we generated both PARP11-knockout (Additional file 1: Fig. S1a-c) and PARP11-overexpressing (Additional file 1: Fig. S1d) A549 cell lines. We found that ZIKV replication was suppressed in PARP11-overexpressing A549 cells as compared with the parental WT A549 cells (Fig. 2a, b). Conversely, ZIKV replication was enhanced in $P A R P 11^{-1-}$ cells (Fig. $2 \mathrm{c}, \mathrm{d}$ ). Furthermore, we monitored the growth kinetics of ZIKV in vector control, PARP11-overexpressing, WT and PARP $11^{-/-}$A549 cells. The results also showed that the ZIKV replication rate was decreased in PARP11-overexpressing cells and was increased in $P A R P 11^{-/}$cells (Fig. 2e, f). Therefore, PARP11 is an anti-ZIKV ISG that may play an important role in innate immune defense against ZIKV replication.

\section{PARP11 suppresses ZIKV independent on the regulation of IFNAR1 protein level}

A recent report indicated that PARP11 inhibited the interferon response by reducing the protein level of IFNAR1 [26] to promote VSV and HSV-1 replication.

In our screening and validation results, however, we found that PARP11 suppressed ZIKV replication 


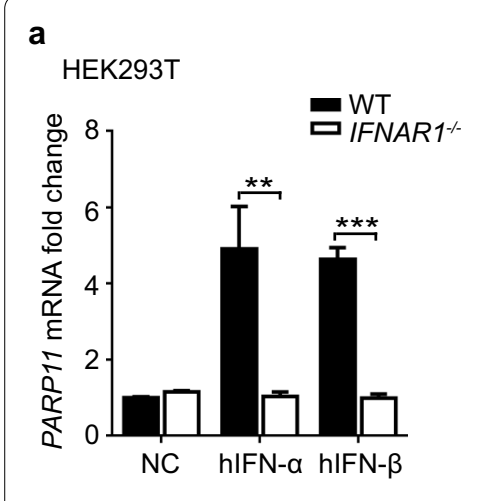

C HEK293T $_{\text {NC } \frac{\text { WT }}{12 \mathrm{~h} 24 \mathrm{~h}} \frac{\mathrm{hIFN}-\beta}{12 \mathrm{~h} 24 \mathrm{~h}}} \frac{\text { NCNAR1\% }}{\frac{\mathrm{hIFN}-\alpha}{12 \mathrm{~h} 24 \mathrm{~h}} \frac{\mathrm{hIFN}-\beta}{12 \mathrm{~h} 24 \mathrm{~h}}}$

b $\quad$ A549

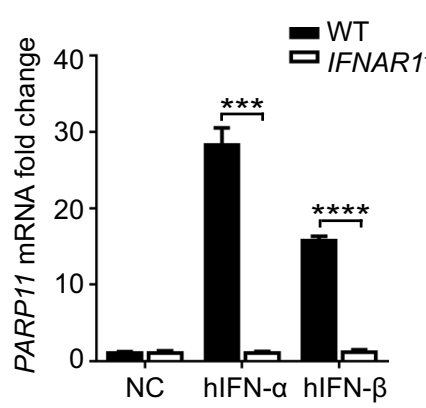

d $_{\text {A549 }}$

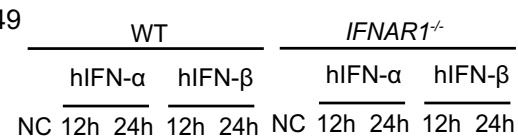

NC $\overline{12 h ~ 24 h} \overline{12 h ~ 24 h}$ NC $\overline{12 h ~ 24 h} \overline{12 h ~ 24 h}$ g A549

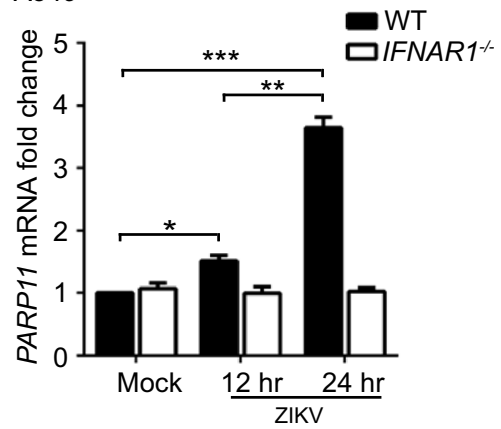

h $\mathbf{A} 549$

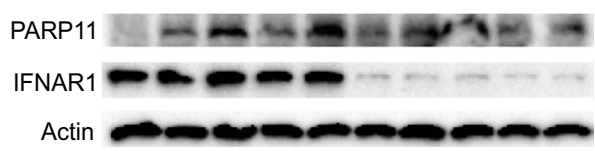

e

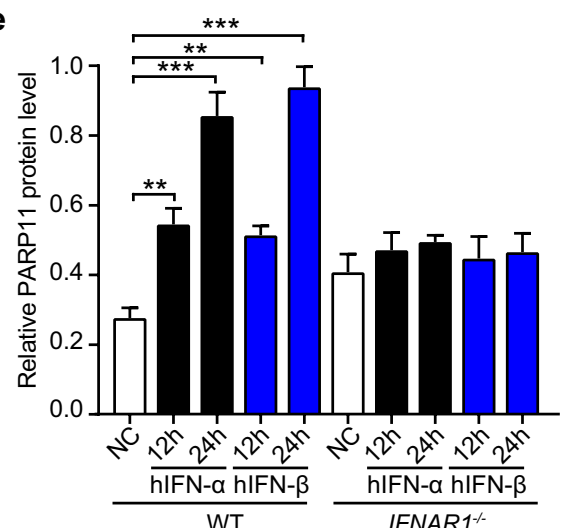

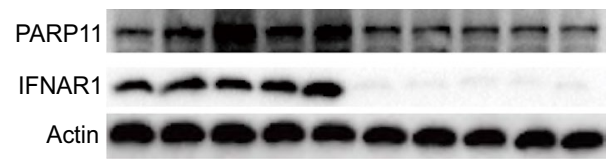

f

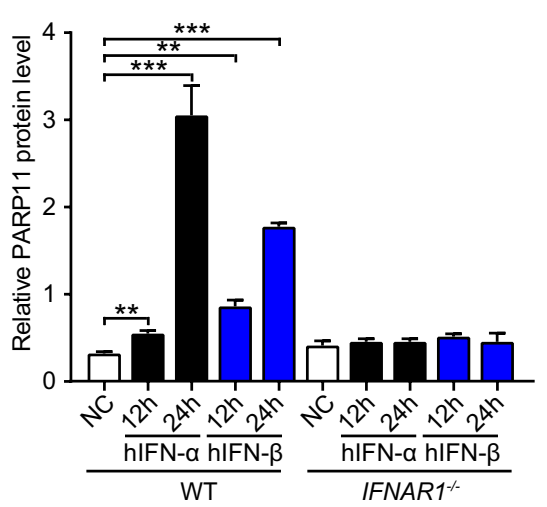

$\frac{\text { WT }}{\text { ZIKV }} \frac{\text { IFNAR } 1^{-1}}{\text { ZIKV }}$

Mock $\overline{24 h 48 h}$ Mock $\overline{24 h ~ 48 h}$

Fig.1 The expression of PARP11 can be induced by IFN and ZIKV infection. a, b qRT-PCR analysis of PARP11 expression in WT and IFNAR ${ }^{-1-}$ HEK293T (a) or A549 (b) cells treated with recombinant human IFN-a (1000 U/mL), IFN- $\beta$ ( 20 ng/mL) and control for 24 h. c, d Western blotting analysis of PARP11 expression in WT and IFNAR1 ${ }^{-/-}$HEK293T (c) or A549 (d) cells treated with recombinant human IFN-a (1000 U/mL), IFN- $\beta$ (20 ng/ $\mathrm{mL}$ ) and control for 12 and $24 \mathrm{~h}$. e Densitometry analysis of the data in (c). $\mathbf{f}$ Densitometry analysis of the data in (d). $\mathbf{g}$ qRT-PCR analysis of PARP11 expression in WT and IFNAR1 ${ }^{-1-}$ A549 cells infected with ZIKV for the indicated times. $\mathbf{h}$ Western blotting analysis of PARP11 expression in WT and IFNAR1 ${ }^{-1-}$ A549 cells infected with ZIKV for the indicated times. i Densitometry analysis of the data in (h). qRT-PCR (a, $\mathbf{b}$ and $\left.\mathbf{g}\right)$ and densitometry $(\mathbf{e}, \mathbf{f}$ and $\mathbf{i})$ data are mean \pm SEM from three independent experiments. Western blotting results $(\mathbf{c}, \mathbf{d}$ and $\mathbf{h})$ are representative images of three independent experiments. ${ }^{*} P<0.05,{ }^{* *} P<0.01,{ }^{* *} P<0.001$ and ${ }^{* * *} P<0.0001$ by Student's $t$ test

(Fig. 2a-f). To test whether PARP11 suppressed ZIKV by regulating protein level of IFNAR1, we infected WT A549 cells with VSV and ZIKV and detected protein level of IFNAR1 by Western blotting. The result showed that ZIKV infection did not obviously change IFNAR1 protein level, while VSV infection decreased IFNAR1 protein level significantly (Fig. 3a, b). We also compared the protein levels of IFNAR1 in WT and PARP11 ${ }^{-1}$ A549 cells infected with VSV and ZIKV. While significant IFNAR1 downregulation was observed in WT and PARP11 A549 cells in response to VSV infection, the IFNAR1 protein level showed no obviously change in both WT and PARP11 $1^{-/}$A549 cells upon ZIKV infection (Fig. 3c, d). On the other hand, we detected a strong increase in protein ADP-ribosylation in WT but not PARP11 ${ }^{-/-}$cells upon ZIKV infection (Fig. 3e, f). These results indicate that PARP11 may suppress ZIKV replication by inducing either host or viral protein ADP-ribosylation in ZIKV 


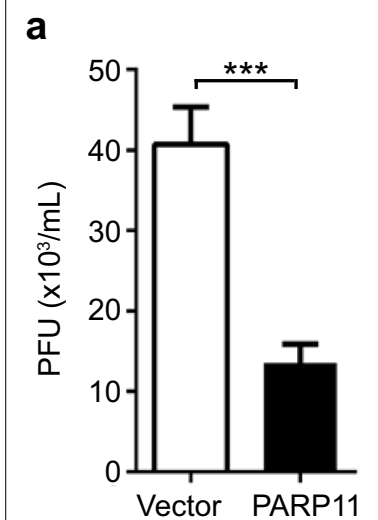

C

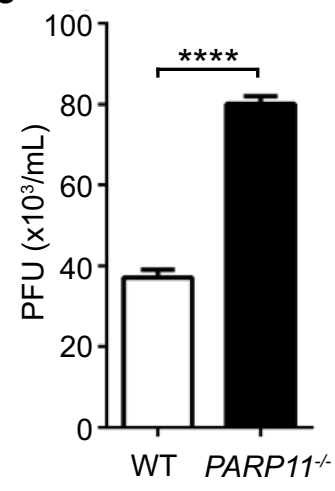

b

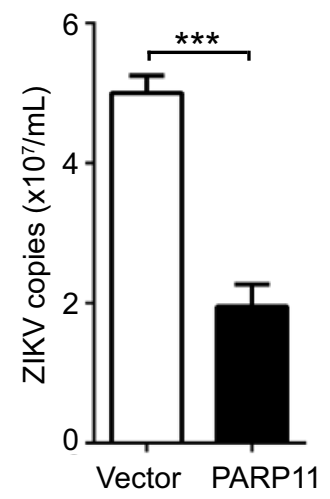

d

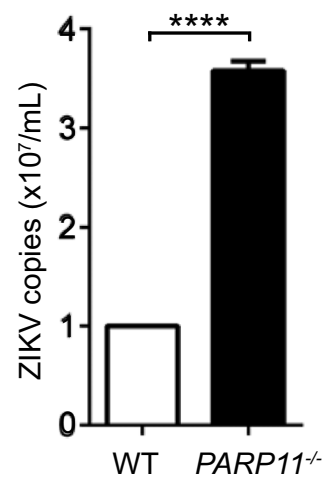

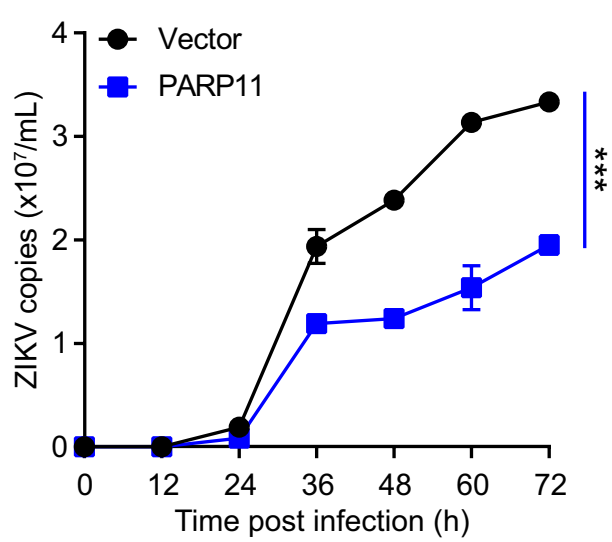

f

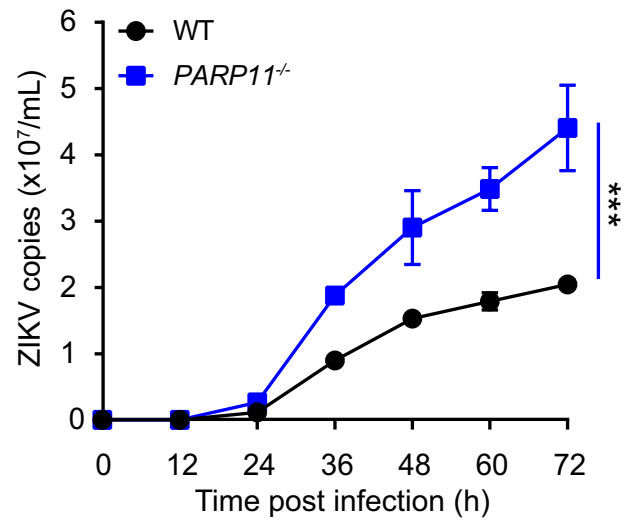

Fig. 2 PARP11 suppresses ZIKV replication in vitro. a, b PARP11-overexpressing or control vector-transfected A549 cells were infected with ZIKV. Viral accumulation after $48 \mathrm{~h}$ in the culture supernatants (a) and cell lysates (b) were measured by plaque assay (a) or qRT-PCR (b). PFU, plaque-forming unites. $\mathbf{c}, \mathbf{d}$ WT or PARP11 $1^{-1-}$ A549 cells were infected with ZIKV. Viral accumulation after $48 \mathrm{~h}$ in the culture supernatants (c) and cell lysates (d) were measured by plaque assay (c) or qRT-PCR (d). e, f Growth curve of ZIKV in PARP11-overexpressing or control vector-transfected A549 cells (e) and WT or PARP11 ${ }^{-1-}$ A549 cells (f). QRT-PCR and viral titers data $(\mathbf{a}-\mathbf{f})$ are mean \pm SEM from three independent experiments. ${ }^{* * *} P<0.001$ and ${ }^{* * *} P<0.0001$ by Student's $t$ test

infected cells instead of regulating the IFNAR1 protein level.

\section{PARP11 suppresses ZIKV independent on its PARP enzyme activity}

PARP11 contains WWE domain at the $\mathrm{N}$ terminus and PARP domain at the $\mathrm{C}$ terminus. The WWE domain is a common interaction module that participates in both ubiquitination and ADP-ribosylation [27]. The PARP domain contains the PARP enzyme activity, which mediates the posttranslational modification of target proteins $[28,29]$. The $\mathrm{Gly}^{205}$ within the PARP domain of human PARP11 binds the amide group of $\mathrm{NAD}^{+}$, which is essential for PARP enzyme activity. To identify whether the PARP enzyme activity of PARP11 is responsible for the anti-ZIKV function, we constructed PARP11 deletion and PARP enzyme activity lost (PARP11 G205A) mutants (Fig. 4a). The expression and expected protein size of these mutants were verified by Western blotting (Fig. 4b).
HeLa cells were transfected with these mutants and subsequently infected by ZIKV to determine how the different domains and PARP enzyme activity affected viral infection. Full-length WT or enzyme inactive PARP11 strongly suppressed ZIKV replication, whereas PARP11 deletion mutants lacking the WWE domain or the PARP domain showed no suppression on ZIKV replication (Fig. 4c). These results indicate that PARP11 suppresses ZIKV replication independent on its PARP enzyme activity.

\section{PARP11 promotes PARP12-mediated ZIKV NS1 and NS3 protein degradation}

In our previous work, we identified PARP12 suppressed ZIKV through PARP-dependent degradation of NS1 and NS3 viral protein. The PARP enzyme activity of PARP12 is essential for ZIKV suppression. However, in this work, we found that PARP11 suppressed ZIKV independent on its PARP enzyme activity 

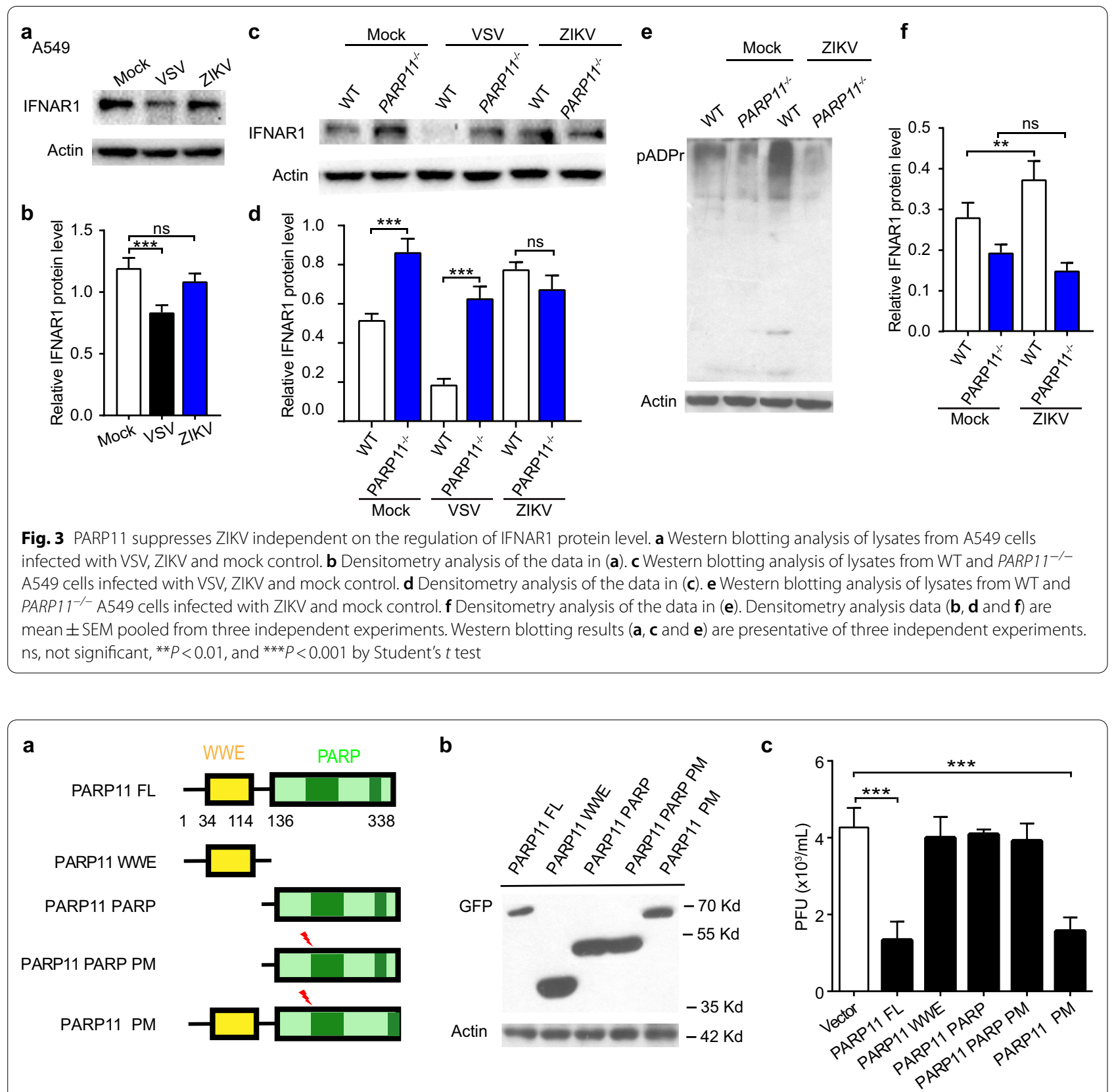

Fig. 4 PARP11 suppresses ZIKV independent on its PARP enzyme activity. a Sketch map of the functional regions of full length (FL) PARP11 and mutant constructs. FL: full length; WWE: WWE domain; PARP: Poly (ADP-ribose) polymerase domain. PM: Point mutation to inactivate PARP enzyme activity. $\mathbf{b}$ Western blotting analysis of lysates from HEK293T cells transfected with the plasmids encoding GFP-tagged FL and mutant PARP11 constructs. c HeLa cells were transfected with FL PARP1 1 and mutant constructs and infected with ZIKV $24 \mathrm{~h}$ after transfection. $48 \mathrm{~h}$ post infection, ZIKV titers in HeLa cells culture supernatant were measured by plaque assay. Western blotting results $(\mathbf{b})$ are presentative of three independent experiments. Viral titers (c) are mean \pm SEM from three independent experiments. ${ }^{* *} P<0.001$ by Student's $t$ test

but can regulate protein ADP-ribosylation in ZIKV infected host cells. To test the relationship between PARP11 and PARP12, we co-transfected His-NS1 and NS3 with HA-PARP12, YFP-PARP11 or Flag-PARP13 as control into WT, PARP $11^{-/-}, P A R P 12^{-/-}$and
PARP11 ${ }^{-/}$PARP12 ${ }^{-/-}$HEK293T cells. Compared to PARP12 co-transfected with vector group, PARP12 co-transfection with PARP11 but not PARP13 further reduced the abundance of NS1 and NS3 proteins (Fig. 5a, b). We also detected the impacts of PARP11 on 


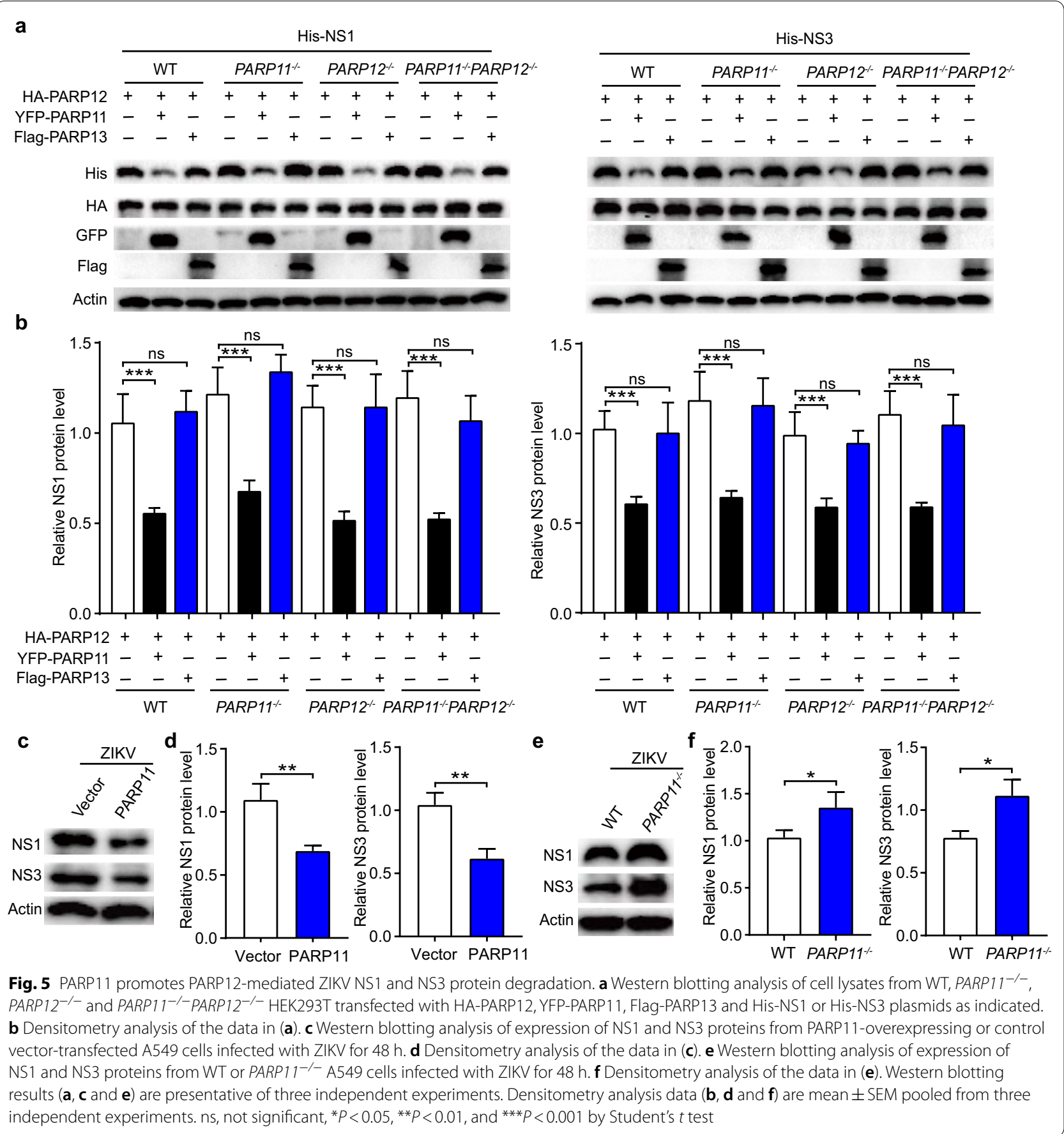

the ZIKV-encoded NS1 and NS3 protein levels in ZIKV infected cells and found that the endogenous NS1 and NS3 protein levels were decreased in PARP11-overexpressed cells and were increased in PARP11 ${ }^{-/}$cells (Fig. 5c-f). These results indicates that PARP11 can cooperate with PARP12 in -mediating ZIKV NS1 and NS3 degradation and may play an important role in controlling the levels of NS1 and NS3 in ZIKV infected cells.

PARP11 promotes PARP12-mediated NS1 and NS3 degradation independent on its PARP enzyme activity To further confirm the involvement of PARP11 in PARP12-mediated NS1 and NS3 degradation, we 
co-transfected His-NS1 or NS3 with increasing amount of HA-PARP12 plasmids in WT, PARP11 ${ }^{-1-}$, and PARP12 ${ }^{-1-}$ HEK293T cells. Compared to WT HEK293T cells, PARP12 showed weaker ability to degrade NS1 and NS3 in PARP11 ${ }^{-1-}$ HEK293T cells (Fig. 6a, b). When PARP11 was transfected back, NS1 and NS3 degradations mediated by PARP12 were increased (Fig. 6c, d). We also examined the PAPR11 mutants on NS1 and NS3 degradation when co-transfected with HA-PARP12 into WT, PARP $11^{-/-}$, PARP $12^{-/-}$and PARP $11^{-/-}$PARP $12^{-/-}$ HEK293T cells. Only the full-length WT and PARP enzyme lost mutation enhanced NS1 and NS3 degradation in synergy with PARP12, PARP11 deletion mutants lacking the WWE domain or the PARP domain did not show impact on enhancing PARP12-mediated NS1 and NS3 degradation (Fig. 6e, f). Interestingly, we observed that PARP11 enzyme lost mutant degraded NS1 and NS3 less efficient than WT PARP11 in PARP11 ${ }^{-/}$HEK293T cells (Fig. 6e, f). Together, these results show that PARP11 promotes the degradation of ZIKV NS1 and NS3 proteins in synergy with PARP12.

\section{PARP11 suppresses ZIKV replication mostly dependent on PARP12}

To further determine whether PARP11 suppressed ZIKV dependent upon PARP12, we compared the replication of ZIKV in WT and PARP12 ${ }^{-/-}$A549 cells in the presence or absence of PARP11 over expression. The results showed that although ZIKV replication was reduced in both WT and PARP12 $2^{-/-}$A549 cells upon PARP11 overexpression PARP11 seemed to be more effective in inhibiting ZIKV replication in PARP12 ${ }^{-/-}$ A549 cells as compared with WT A549 cells (Fig. 7a-c). We then checked whether the degradations of NS1 and NS3 mediated by PARP11 WT and PARP11 enzyme lost mutant were also impacted by PARP12. Western blot analysis revealed that PARP11 WT and PARP11 enzyme lost mutant can still degrade NS1 and NS3 in PARP12 $2^{-1}$ HEK293T cells but at an efficiency significantly lower than that in WT HEK293T cells (Fig. 7d-g). These results suggest that PARP11 suppresses NS1 and
NS3 degradation and ZIKV replication mostly dependent on the existence of PARP12.

\section{PARP11 interacts and co-localizes with PARP12}

To elucidate the mechanism by which PARP11 suppressed ZIKV in cooperation with PARP12, we firstly examined the interaction between PARP11 and PARP12. We demonstrated that PARP11 could interact and colocalize with PARP12 protein by co-immunoprecipitation and immunofluorescence assay (Fig. 8a, b). To further identify the protein module of PARP11 that interacted with PARP12, we co-transfected HEK293T cells with HA-PARP12 and EGFP-PARP11 WWE and PARP domain expressing plasmids and performed coimmunoprecipitation assay with HA-tagged agarose beads. Full-length PARP12 interacted with the WWE domain of PARP11 but not the PARP domain (Fig. 8c). This result is in line with the function of WWE domain as a common interaction module that participates in both ubiquitination and ADP-ribosylation. We further constructed HA tagged PARP12 mutant which expressed ZnF, WWE and PARP domain of PARP12 (Fig. 8d), and performed immunoprecipitation assay to examine which domain of PARP12 interacted with PARP11. The result indicates that PARP12 interacts with PARP11 through its WWE domain (Fig. 8e). We then performed immunoprecipitation assay to examine which domain of PARP12 interacted with ZIKV NS1 protein, and found that PARP12 interacted with NS1 through its PARP domain (Fig. 8f). These results demonstrate that PARP11 and PARP12 interact and cooperate with each other on ZIKV suppression.

\section{Discussion}

Although the ZIKV outbreak happened in 2015-2017, ZIKV continues to develop and evolve in a form of asymptomatic infectious. According to a previous report, researchers firstly detected the appearance of the ZIKV Africa lineage in Brazil, indicating the risk of a new epidemic [6]. Research on the pathology of ZIKV to explore for available means on epidemic control and associated disease treatment is still urgently needed. In our

\footnotetext{
(See figure on next page.)

Fig. 6 PARP11 promotes PARP12-mediated NS1 and NS3 degradation independent on its PARP enzyme activity. a Western blotting analysis of cell lysates from WT, PARP11 1 $1^{--}$and PARP12 $2^{-1-}$ HEK293T transfected with His-NS1 or NS3 and increasing amounts of HA-PARP12 plasmids (0, 250, 500 ng). b Densitometry analysis of the data in (a). c Western blotting analysis of cell lysates from WT, PARP11 ${ }^{-/-}$and PARP12 $2^{-/}$HEK293T transfected with HA-PARP12, YFP-PARP11 and His-NS1 or His-NS3 plasmids. $\mathbf{d}$ Densitometry analysis of the data in (c). e Western blotting analysis of cell lysates

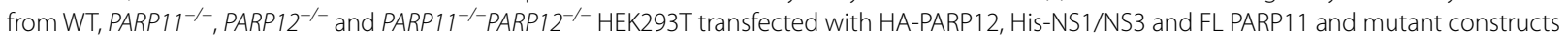
plasmids. FL: full length; WWE: WWE domain; PARP: Poly (ADP-ribose) polymerase domain. PARP PM: PARP domain with point mutation to inactivate PARP enzyme activity. PM: PARP11 full length protein with point mutation to inactivate PARP enzyme activity. $\mathbf{f}$ Densitometry analysis of the data in (e). Western blotting results ( $\mathbf{a}, \mathbf{c}$ and $\mathbf{e})$ are presentative of three independent experiments. Densitometry analysis data $(\mathbf{b}, \mathbf{d}$ and $\mathbf{f})$ are mean $\pm S E M$ pooled from three independent experiments. ns, not significant, ${ }^{*} P<0.05,{ }^{* *} P<0.01,{ }^{* * *} P<0.001$, and ${ }^{* * *} P<0.0001$ by Student's $t$ test.
} 
a

\begin{tabular}{l} 
His-NS1 \\
WT \\
\hline PARP11
\end{tabular}
HA-PARP12 - + ++ + ++ - +++ -+++-+++-+++ His

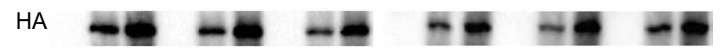
Actin -

b

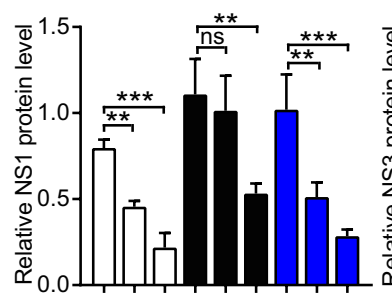
HA-PARP12

$$
\frac{\frac{-+++}{\text { WT }} \frac{-+++}{\text { PARP11-/ }} \frac{-+++}{\text { PARP12/- }}}{\text { His-NS1 }}
$$

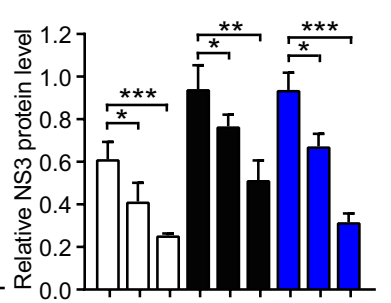

C

$\frac{\text { His-NS1 }}{\text { WT } \text { PARP11- PARP12- }}$

His-NS3

WT PARP11\% PARP12 HA-PARP12 - + $++\overline{-+} \overline{-+} \overline{-++} \overline{-+}$ YFP-PARP11 -

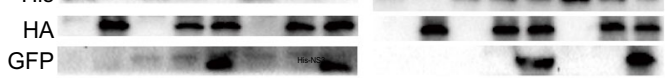

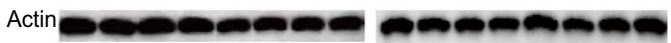

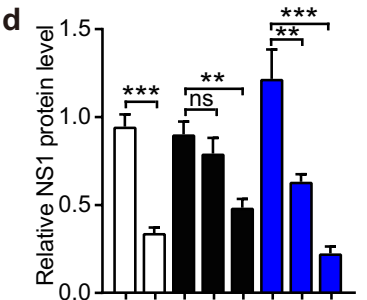

HA-PARP $12-+++++$ 1 WTP $\frac{--+}{-1}-\frac{-+}{\text { PARP } 12}$

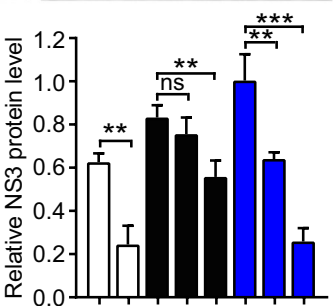

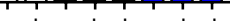
$-+-++++$ $\overline{\text { WTPARP11 }} \overline{\text { PARP12 }}$

e

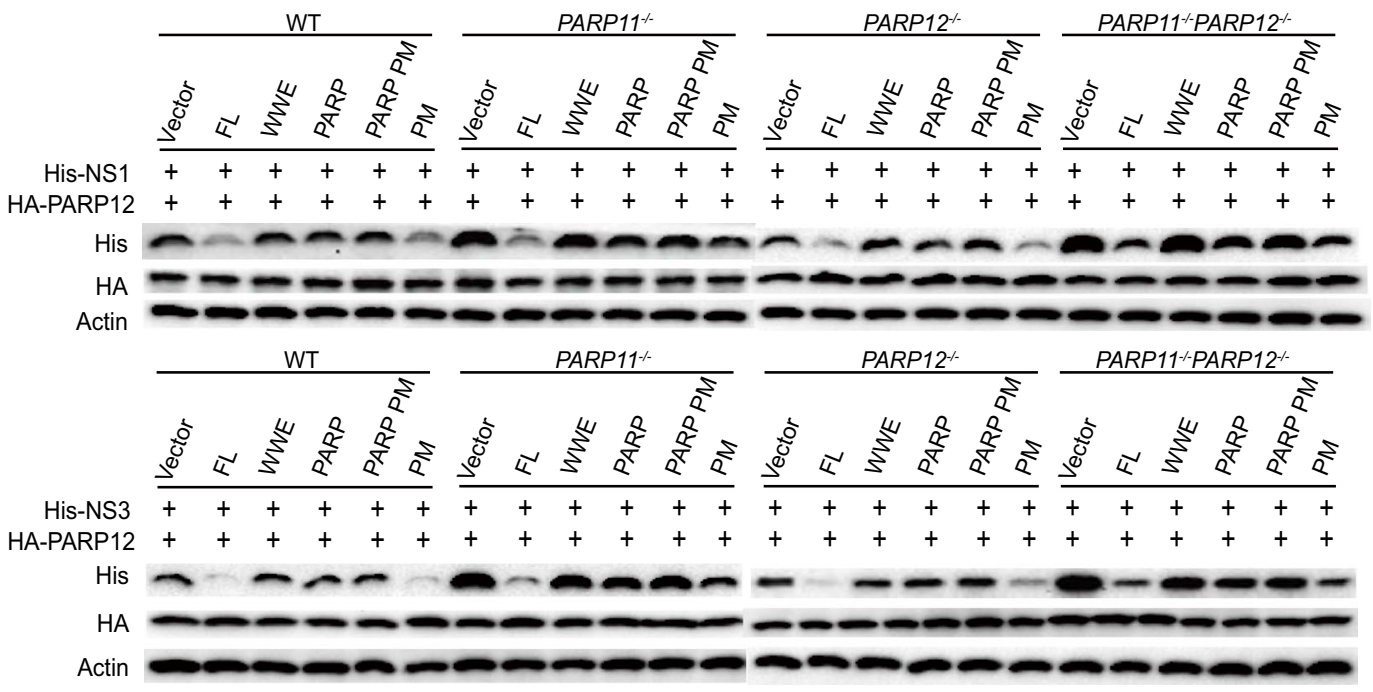

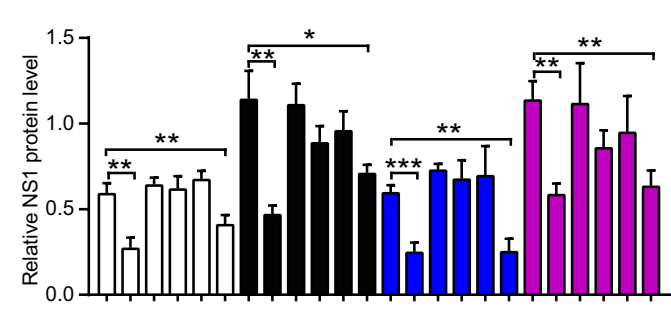

His-NS1 HA-PARP12

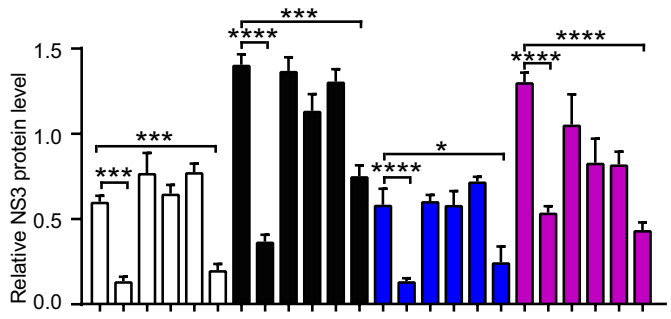

His-NS3 ++++++++++++++++++++++++ HA-PARP12

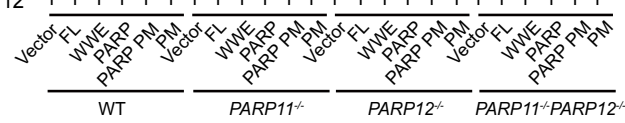




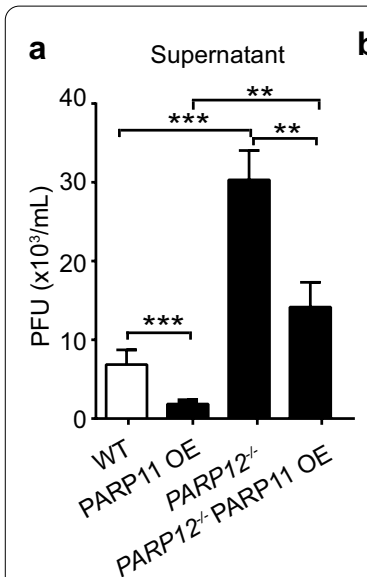

d

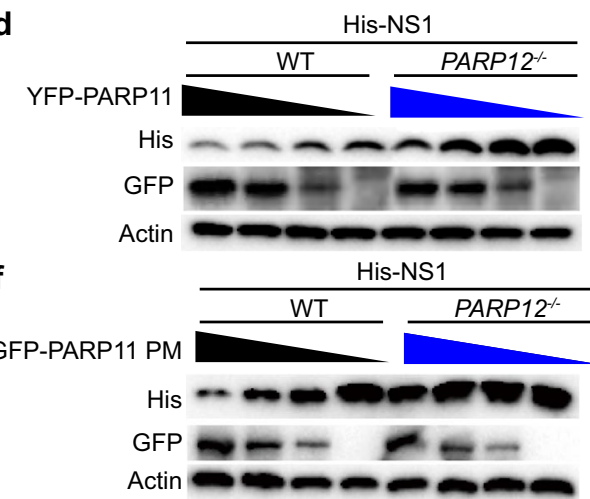

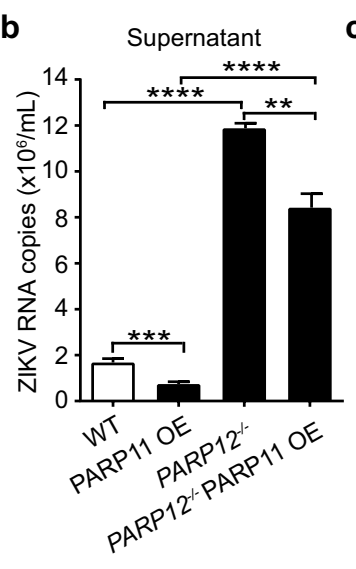
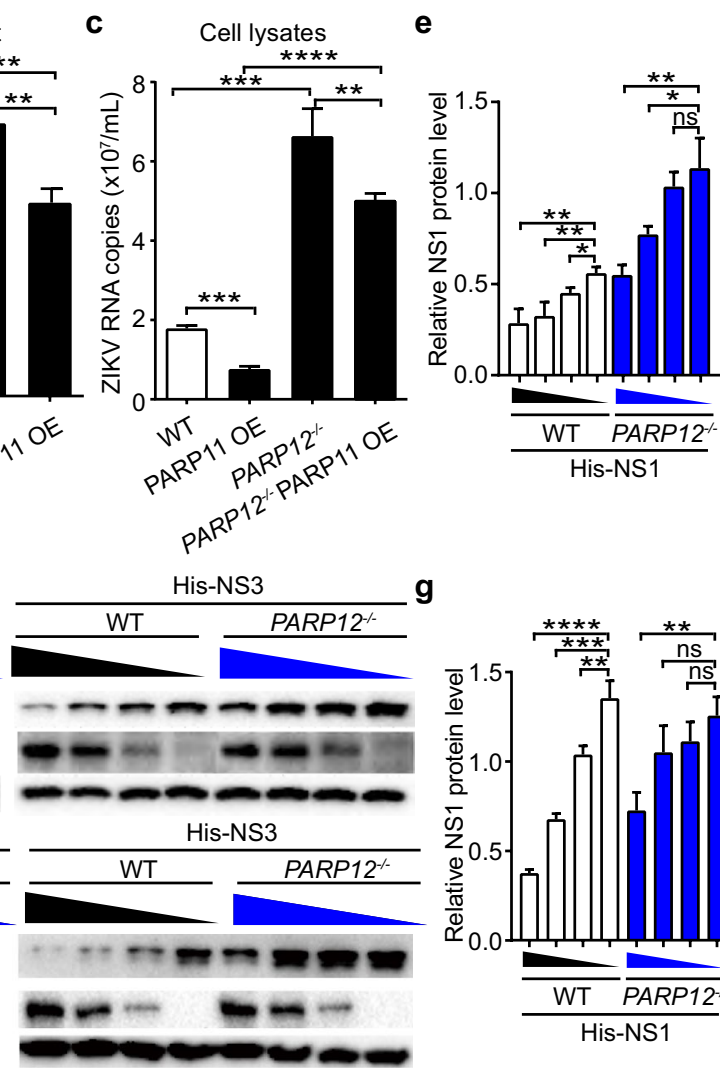

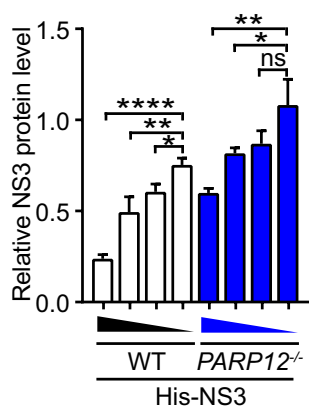

Fig. 7 PARP1 1 suppresses ZIKV mostly dependent on PARP12. a-c PARP11-overexpressing or control vector-transfected WT and PARP12 ${ }^{-/-}$A549 cells were infected with ZIKV. Viral accumulation after $48 \mathrm{~h}$ in the culture supernatants (a and $\mathbf{b})$ and cell lysates (c) were measured by plaque assay (a) or qRT-PCR (b and $\mathbf{c}$ ). PFU, plaque-forming unites. d Western blotting analysis of cell lysates of cell lysates from WT and PARP12 ${ }^{-1-}$ HEK293T cells transfected with His-NS1 or NS3 and decreasing amounts of YFP-PARP11 plasmids (500, 300, 100, 0 ng). e Densitometry analysis of the data in (d). f Western blotting analysis of cell lysates of cell lysates from WT and PARP12 ${ }^{-/}$HEK293T cells transfected with His-NS1 or NS3 and decreasing amounts of YFP-PARP1 1 enzyme lost mutant plasmids (500, 300, 100, 0 ng). $\mathbf{g}$ Densitometry analysis of the data in (f). qRT-PCR, viral titers and densitometry analysis data $(\mathbf{a}-\mathbf{c}, \mathbf{e}$ and $\mathbf{g})$ are mean \pm SEM from three independent experiments. Western blotting results $(\mathbf{d}$ and $\mathbf{f})$ are presentative of three independent experiments. ${ }^{* *} P<0.01,{ }^{* * *} P<0.001$ and ${ }^{* * * *} P<0.0001$ by Student's $t$ test

previously work, we identified PARP12 with anti-ZIKV function and the detailed mechanism was provided [18]. Here, we found that PARP11 suppresses ZIKV in cooperated with PARP12 (Fig. 5a-f). Our studies describe a novel mechanism of PARP11 in viral restriction that is quite different from the previous report indicating that PARP11 promotes VSV and HSV replication by degrading IFNAR1 and inhibiting IFN signaling pathway [26] as we observed no significant change of IFANR1 at protein level in ZIKV infected A549 WT and PARP11-deficency cells (Fig. 3a-d). We speculated the different function of PARP11 may be a result from the characteristic of different virus species.

In this work, we identified PARP11 as a new antiZIKV ISG that suppresses ZIKV replication in cooperation with PARP12. Several PAPRs have been found with anti-viral functions dependent or independent on their PARP enzyme activities. PARP12, for an example, can utilize its PARP enzyme activity to poly ADP-ribosylate ZIKV NS1 and NS3 proteins which promotes their subsequently ubiquitination and degradation by proteasome [18]. PARP13, also known as ZnF antiviral protein (ZAP), inhibits various viruses by directly degrading viral mRNA or protein independent of its PARP enzyme activity [30]. PARP11 was reported as a novel enzyme important for proper sperm head shaping and a potential factor involved in idiopathic mammalian teratozoospermia. In this process PARP11 exhibits mono ADP-ribosylation activity which ADP-ribosylates itself and is essential for co-localization of PARP11 with the nuclear pore components [31]. Other research also indicated that the cellular location of PARP11 is regulated by its PARP catalytic activity [32]. In this work, we found that PARP11 suppresses ZIKV independent on its PARP enzyme activity (Fig. $4 \mathrm{a}-\mathrm{c}$ ) but is still involved in increased protein ADPribosylation in ZIKV infected host cells (Fig. 3e, f). We 


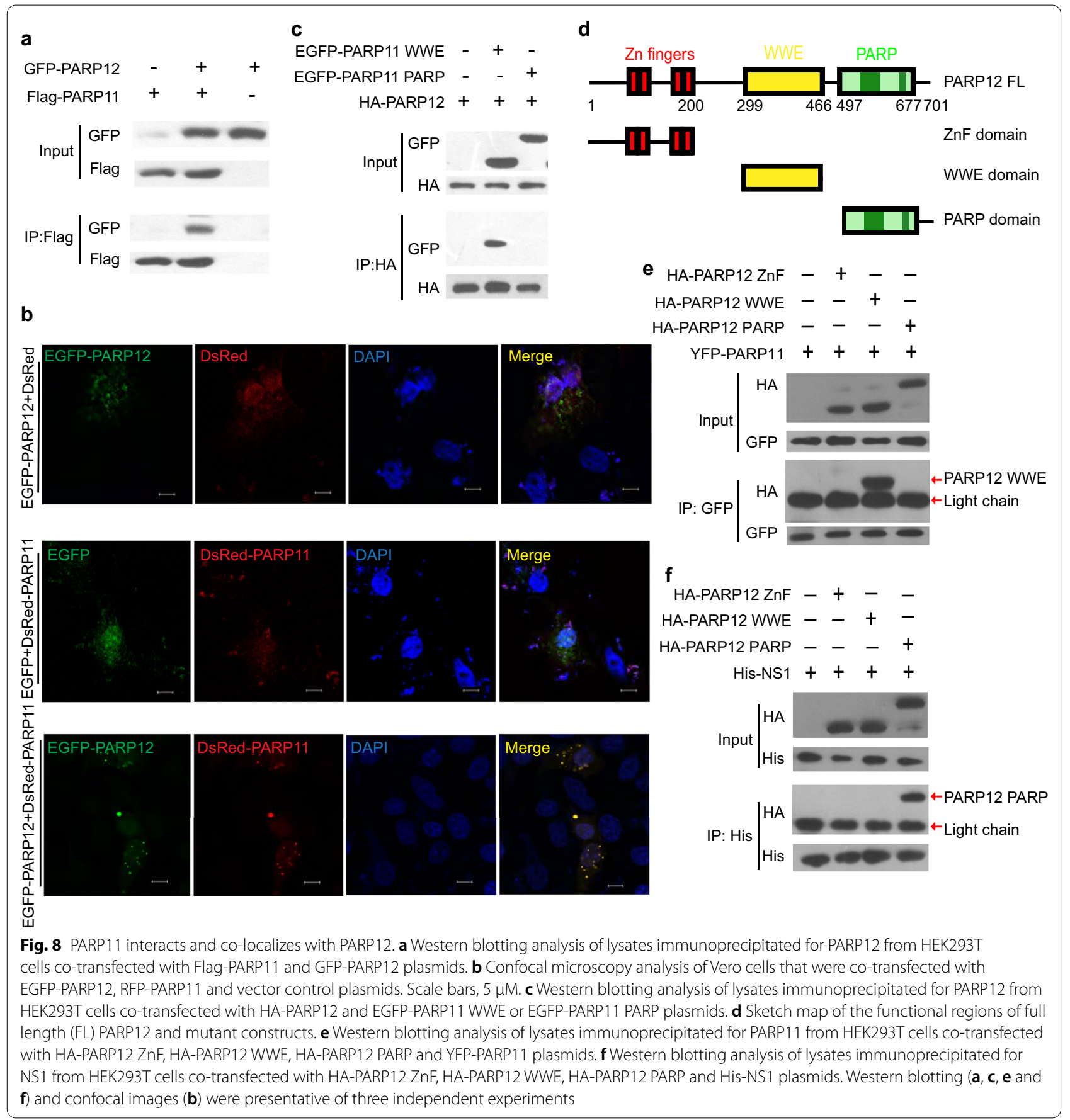

showed that the existence of PARP11 enhances the NS1 and NS3 degradation mediated by PARP12. In addition, the anti-viral function of PARP11 was largely impaired in the absence of PARP12, indicating that PARP11 inhibits ZIKV replication dependent on the existence of PARP12 (Fig. $7 \mathrm{a}-\mathrm{c}$ ). These results suggest that PARP11 cooperates with PARP12 in ZIKV protein degradation through enhancing NS1 and NS3 degradation. We also noticed that PARP11 can suppress ZIKV replication and NS1 and NS3 protein degradation in the absence of PARP12, although at a much lower efficiency than that in the presence of PARP12 (Fig. $7 \mathrm{~d}-\mathrm{g}$ ). So, other unrevealed mechanism might be involved in the antiviral activity of PARP11 independent of PARP12. Thus, more attention should be paid to the anti-viral role of PARP family proteins, especially PARP11 and PARP12. 
We further identified that the WWE domains of both PARP11 and PARP12 are involved in their interaction (Fig. 8c). Considering that PARP12 ADP-ribosylates NS1 and NS3 dependent on its PARP enzyme activity, PARP12 may work as an intermediate that interacts with NS1 and NS3 proteins by its PARP domain and interacts with PARP11 by its WWE domain. PARP11, NS1 and NS3 proteins, and PARP12 constitute a degradation complex in which PARP11 assists PARP12-mediated ADPribosylation in an unknown mechanism.

In conclusion, our work highlights a novel anti-ZIKV role of PARP11 and its mechanism responsible for enhancing PARP-12-mediated NS1 and NS3 degradation. As more and more PARP inhibitors have been developed, further studies on the antiviral activities of different PARP family members will likely provide additional treatments for diseases associated with viruses such as ZIKV.

\section{Materials and methods Virus and cells}

ZIKV strain GZ01/2016 (Genbank Accession Number KU820898) and VSV virus was used at a multiplicity on infection (MOI) of 0.1 in this study, except where indicated otherwise [33]. The IFNAR1 ${ }^{-/-}$HEK293T and A549 cell lines were generated as described [19]. A549, BHK-21, Vero, HeLa and HEK293T cells were purchased from America Type Culture Collection and cultured in Dulbecco's modified Eagle's medium (DMEM) $\left(37^{\circ} \mathrm{C}, 5 \%\right.$ $\mathrm{CO}_{2}$ ) supplemented with $10 \%$ fetal bovine serum (FBS), $100 \mathrm{U} / \mathrm{mL}$ penicillin and $50 \mu \mathrm{g} / \mathrm{mL}$ streptomycin.

\section{Plaque assay}

BHK-21 cells were seeded in a 12-well plate for $12 \mathrm{~h}$. Cells were washed with PBS once and infected with virus samples for $1 \mathrm{~h}$. The culture supernatant was aspirated and replaced with DMEM containing 1\% low-melting agarose and $2 \%$ FBS. Viral plaques were stained and counted 4 days after infection. The titer of ZIKV was quantified by plaque assay and normalized to control.

\section{DNA constructs and stable cell line generation}

pMOI-GFP and pMOI-PARP11 (Homo sapiens) expression plasmids were purchased from GeneCopoeia and described previously [34]. The viral RNA of the GZ01/2016 strain was isolated and used in reverse transcription PCR experiments to obtain the complementary DNA (cDNA) sequence of ZIKV nonstructural NS1 and NS3 proteins. ZIKV NS1 and NS3 genes were cloned into the pcDNA6/V5-His expression vector (Invitrogen) using standard molecular techniques and verified by sequencing. DsRed-PARP11, EGFP-PARP12, FlagPARP11, HA-PARP12, HA-PARP12 ZnF, HA-PARP12 WWE, HA-PARP12 PARP, EGFP-PARP11 WWE domain, EGFP-PARP11 PARP domain, YFP-PARP11, Flag-PARP13 and GFP-PARP11 mutants were cloned using standard molecular cloning and oligonucleotide mutagenesis methods. To create a stable cell line for PARP11 expression, PARP11 was cloned into the pMXsIG-IgkFLAG vector and co-transfected into HEK293T cells with VSV glycoprotein and pCpG helper plasmids. $48 \mathrm{~h}$ after transfection, the culture supernatant was collected and added into WT or PARP12 $12^{-/-}$A549 cells for infection. The cells were collected $72 \mathrm{~h}$ after infection, and the PARP11-overexpressing cells were then sorted by fluorescence-activated cell sorting (FACS).

\section{Western blotting}

All cells were treated as indicated and lysed with lysis buffer [50 mM tris- $\mathrm{HCl}$ (pH 7.5), $150 \mathrm{mM} \mathrm{NaCl}, 5 \mathrm{mM}$ EDTA, 1\% NP-40, $1 \mathrm{mM}$ PMSF, and $1 \times$ protein inhibitor (Roche)]. The cell extracts were immunoblotted with the indicated antibodies to measure the level of the expressed proteins. Mouse anti- $\beta$-actin (ZSGB-Bio), rabbit antiGFP (Abcam), rabbit anti-PARP11 (Finetest), rabbit anti-IFNAR1(Abcam), mouse anti-poly (ADP-ribose) (GeneTex), rabbit anti ZIKV NS1 (Genetex), rabbit antiZIKV NS3 (Genetex), mouse anti-HA, mouse anti-His, and mouse anti-Flag tag antibodies (Sigma-Aldrich) were used for detection at the appreciated dilutions.

\section{Co-immunoprecipitation assay}

HEK293T cells were transfected with the indicated plasmids. $30 \mathrm{~h}$ after transfection, protein was extracted using solution A [50 mM tris- $\mathrm{HCl}(\mathrm{pH} 7.5), 150 \mathrm{mM}$ $\mathrm{NaCl}, 5 \mathrm{mM}$ EDTA, 1\% Triton-X100, $1 \mathrm{mM}$ phenylmethylsulfonylfluoride (PMSF), and $1 \times$ protein inhibitor (Roche)]. An aliquot of the extracts was immunoblotted with the indicated antibodies. The remaining extracts were immunoprecipitated using Sepharose beads bound to anti-Flag, anti-HA, anti-His or anti-GFP antibodies (Sigma-Aldrich) at $4{ }^{\circ} \mathrm{C}$ overnight. After washing the Sepharose beads four times with solution B [50 mM tris- $\mathrm{HCl}$ (pH 7.5), $150 \mathrm{mM} \mathrm{NaCl}, 5 \mathrm{mM}$ EDTA, $0.2 \%$ Triton-X100, and $1 \mathrm{mM} \mathrm{PMSF}$ ], proteins were eluted by heating the beads to $98{ }^{\circ} \mathrm{C}$ in $1 \times$ SDS-polycrylamid gel electrophoresis loading buffer $[50 \mathrm{mM}$ Tris- $\mathrm{HCl}(\mathrm{pH}$ 6.8), 2\% (V/V) SDS, 6\% (V/V) glycerol, and 2\% (V/V) $\beta$-mercaptoethanol]. The eluted was analyzed by Western blotting with the indicated antibodies.

\section{Gene knockout by the CRISPR/Cas9 system}

To knockout PARP11 and PARP12 in A549 and HEK293T cell lines, two small guide RNAs (SgRNAs) $(\sim 100 \mathrm{bp}$ gap sequence) targeting the PARP11 and PARP12 genes were designed and cloned into sgRNA expression vectors under the control of human U6 promotor. A549 or 
HEK293T cells were transfected with sgRNAs and Cas9 expression plasmids, followed by puromycin selection, as described previously $[35,36]$. Sigle clones were isolated by FACS and confirmed by PCR genotyping and sequencing.

\section{RNA isolation, reverse transcription, and PCR}

Total RNA from cells or viruses was extracted with the PureLink RNA Extraction kit (Thermo Fisher Scientific). Viral RNA copies were measured by qRT-PCR [37] with the One Step PrimeScript RT-PCR kit (Takara). ZIKV primers and TaqMan probes were described previously [38]. Primers used to amplify corresponding genes were obtained from PrimerBank (http://pga.mgh.harvard.edu/ primerbank/). SYRB Green qPCR mix (TransGen Biotec) was used to analyze mRNA levels on an ABI 7500 (Applied Biosystems) analyzer.

\section{Immunofluorescence staining and confocal imaging}

Vero cells were seeded in a confocal dish (Solarbio) and transfected with EGFP-PARP12 and DsRed-PARP11 plasmids. After $24 \mathrm{~h}$, cells were fixed with $0.4 \%$ paraformaldehyde for $15 \mathrm{~min}$ and permeabilized in $0.2 \%$ Triton-X100 for $15 \mathrm{~min}$ at room temperature. The cells were washed three times with PBS supplemented with $0.05 \%$ Tween-20. Nuclei were stained with $4^{\prime} 6$-diamidino-2-phenylindole (Thermo Fisher Scientific). Cells were imaged on a LSM700 (Carl Zeiss) confocal microscope, and the images were analyzed with ImageJ software.

\section{Statistical analysis}

All data were analyzed using Prism software (Graphpad 8.0). Statistical evaluation was performed by twoway Student's $t$ test. Data are mean \pm SEM, and $P$ values are indicated by ns, not significant, ${ }^{*} P<0.05,{ }^{* *} P<0.01$, ${ }^{* * *} P<0.001$ and ${ }^{* * * *} P<0.0001$. All cellular experiments were repeated at least three times.

\author{
Abbreviations \\ PARP: Poly-adenosine 5'-diphosphate (ADP)-ribose polymerases; ZIKV: Zika \\ virus; NS1: Non-structural protein 1; NS3: Non-structural protein 3; IFNAR1: \\ IFNa/ $\beta$ receptor subunit 1; VSV: Vesicular stomatitis virus; IFN: Interferon; ISGs: \\ Interferon-stimulated genes.
}

\section{Supplementary Information}

The online version contains supplementary material available at https://doi. org/10.1186/s13578-021-00628-y.

Additional file 1: Figure S1. Construction of PARP11 knockout and PARP11-overexpressing A549 cell lines. a Design of two sgRNA targeting the genome loci of PARP11 in A549 cells. b Deletion of 100 bp genomic DNA in a PARP $11^{-/-}$clone was confirmed by PCR. c WT and PARP $11^{-/-}$cells were immunoblotted for PARP11. d Verification of PARP11-overexpressing and vector control A549 (GFP tagged) cells were immunoblotted for GFP. Western blotting results (b-d) are representative of three independent experiments.
Acknowledgements

Not applicable.

\section{Authors' contributions}

GC and LL jointly designed this study. LL, SZ, NS, and JL performed plaque assay and GRT-PCR experiments. LL, XX, and LP performed Western blotting, coimmunoprecipitation and confocal assay. YS, CP and LL performed CRISPR/ Cas9 knocked out assay. GC, HY, SL and LL wrote and revised the manuscript. All authors read and approved the final manuscript.

\section{Authors' information}

Lili Li is an associate professor at the Center for Systems Medicine, Institute of Basic Medical Sciences, Chinese Academy of Medical Sciences \& Peking Union Medical College. She has a Ph.D. in cell biology from Chinese Academy of Sciences, and her research interests are anti-viral interferon-stimulated genes and anti-viral innate immunity.

\section{Funding}

This project is financially supported by Chinese Academy of Medical Sciences Innovation Fund for Medical Sciences (2016-12M-1-005 and 2019-12M-1-003), National Science funds of China (82073181, 81802870 and 2017YFA0506200), the Non-profit Central Research Institute Fund of Chinese Academy of Medical Sciences (2020-PT310-006, 2019XK310002 and 3332018131), US National Institute of Health funds (Al069120, Al056154, Al140718, and Al028697), the UCLA AIDS Institute and UCLA David Geffen School of Medicine-Eli and Edythe Broad Center of Regenerative Medicine and Stem Cell Research Award Program. HY is supported by Science funds from Jiangsu Province (BK20170407) and the Innovative and Entrepreneurial Team grant (2018-2021) from Jiangsu Province. LL is supported by the Chinese Postdoctoral Science Foundation (2019M650564) and Innovative and Entrepreneurial Doctor grant (2020-2022) from Jiangsu Province.

\section{Availability of data and materials}

The datasets used or analyzed during the current study are available from the corresponding author on reasonable request.

\section{Declarations}

Ethics approval and consent to participate

Not applicable.

Consent for publication

Written informed consent for publication was obtained from all participants.

\section{Competing interests}

The authors have declared that no conflict of interest exists.

\section{Author details}

${ }^{1}$ Center for Systems Medicine, Institute of Basic Medical Sciences, Chinese Academy of Medical Sciences and Peking Union Medical College, Beijing 100005, China. ${ }^{2}$ Suzhou Institute of Systems Medicine, Suzhou 215123, Jiangsu, China. ${ }^{3}$ Lineberger Comprehensive Cancer Center, University of North Carolina At Chapel Hill, Chapel Hill, NC 27599, USA. ${ }^{4}$ CAS Key Laboratory of Infection and Immunity, Institute of Biophysics, Chinese Academy of Sciences, Chaoyang District, Beijing 100101, China. ${ }^{5}$ Department of Microbiology, Immunology and Molecular Genetics, University of California, Los Angeles, CA 90095, USA.

Received: 3 April 2021 Accepted: 11 June 2021

Published online: 29 June 2021

\section{References}

1. Dick GW, Kitchen SF, Haddow AJ. Zika virus. I. Isolations and serological specificity. Trans R Soc Trop Med Hyg. 1952;46(5):509-20.

2. Singh RK, Dhama K, Malik YS, Ramakrishnan MA, Karthik K, Tiwari R, et al. Zika virus - emergence, evolution, pathology, diagnosis, and control: current global scenario and future perspectives - a comprehensive review. Vet Q. 2016;36(3):150-75. 
3. Sharma V, Sharma M, Dhull D, Sharma Y, Kaushik S, Kaushik S. Zika virus: an emerging challenge to public health worldwide. Can J Microbiol. 2020;66(2):87-98

4. Kazmi SS, Ali W, Bibi N, Nouroz F. A review on Zika virus outbreak, epidemiology, transmission and infection dynamics. J Biol Res (Thessalon). 2020;27:5.

5. Bhardwaj S, Gokhale MD, Mourya DT. Zika virus: current concerns in India. Indian J Med Res. 2017;146(5):572-5.

6. Kasprzykowski J, Fukutani KF, Fabio H, Fukutani ER, Costa LC, Andrade BB, et al. A recursive sub-typing screening surveillance system detects the appearance of the ZIKV African lineage in Brazil: is there a risk of a new epidemic? Int J Infect Dis. 2020;96:579-81.

7. Martins MM, Medronho RA, Cunha A. Zika virus in Brazil and worldwide: a narrative review. Paediatr Int Child Health. 2021;41(1):28-35.

8. Muller WJ, Mulkey SB. Lessons about early neurodevelopment in children exposed to ZIKV in utero. Nat Med. 2019;25(8):1192-3.

9. Nielsen-Saines K, Brasil P, Kerin T, Vasconcelos Z, Gabaglia CR, Damasceno $\mathrm{L}$, et al. Delayed childhood neurodevelopment and neurosensory alterations in the second year of life in a prospective cohort of ZIKV-exposed children. Nat Med. 2019:25(8):1213-7.

10. Ospina ML, Tong VT, Gonzalez M, Valencia D, Mercado M, Gilboa SM, et al. Zika virus disease and pregnancy outcomes in Colombia. N Engl J Med. 2020;383(6):537-45.

11. Rasmussen SA, Jamieson DJ. Teratogen update: Zika virus and pregnancy. Birth Defects Res. 2020;112(15):1139-49.

12. Pattnaik A, Sahoo BR, Pattnaik AK. Current status of Zika virus vaccines: successes and challenges. Vaccines (Basel). 2020;8(2):266.

13. Lunardelli VAS, Apostolico JS, Fernandes ER, Santoro RD. Zika virus-an update on the current efforts for vaccine development. Hum Vaccin Immunother. 2021;17(3):904-8.

14. Bernatchez JA, Tran LT, Li J, Luan Y, Siqueira-Neto JL, Li R. Drugs for the treatment of Zika virus infection. J Med Chem. 2020;63(2):470-89.

15. Xie X, Shan C, Shi PY. Restriction of Zika virus by host innate immunity. Cell Host Microbe. 2016;19(5):566-7.

16. Zhu X, He Z, Yuan J, Wen W, Huang X, Hu Y, et al. IFITM3-containing exosome as a novel mediator for anti-viral response in dengue virus infection. Cell Microbio. 2015;17(1):105-18.

17. Snyder B, Goebel S, Koide F, Ptak R, Kalkeri R. Synergistic antiviral activity of Sofosbuvir and type-l interferons (alpha and beta) against Zika virus. Med Virol. 2018;90(1):8-12.

18. Li L, Zhao H, Liu P, Li C, Quanquin N, Ji X, et al. PARP12 suppresses Zika virus infection through PARP-dependent degradation of NS1 and NS3 viral proteins. Sci Signal. 2018;11(535):eaas9332.

19. Li C, Deng YQ, Wang S, Ma F, Aliyari R, Huang XY, et al. 25-Hydroxycholesterol protects host against Zika virus infection and its associated microcephaly in a mouse model. Immunity. 2017;46(3):446-56.

20. Bock FJ, Chang P. New directions in poly(ADP-ribose) polymerase biology. FEBS J. 2016;283(22):4017-31.

21. Aravind L, Zhang D, de Souza RF, Anand S, Iyer LM. The natural history of ADP-ribosyltransferases and the ADP-ribosylation system. Curr Top Microbiol Immunol. 2015;384:3-32.

22. Rack JGM, Palazzo L, Ahel I. (ADP-ribosyl)hydrolases: structure, function, and biology. Genes Dev. 2020;34(5-6):263-84.
23. Kuny CV, Sullivan CS. Virus-host interactions and the ARTD/PARP family of enzymes. PLoS Pathog. 2016;12(3):e1005453.

24. Daugherty MD, Young JM, Kerns JA, Malik HS. Rapid evolution of PARP genes suggests a broad role for ADP-ribosylation in host-virus conflicts. PLoS Genet. 2014;10(5):e1004403.

25. Fehr AR, Singh SA, Kerr CM, Mukai S, Higashi H, Aikawa M. The impact of PARPs and ADP-ribosylation on inflammation and host-pathogen interactions. Genes Dev. 2020;34(5-6):341-59.

26. Guo T, Zuo Y, Oian L, Liu J, Yuan Y, Xu K, et al. ADP-ribosyltransferase PARP11 modulates the interferon antiviral response by monoADP-ribosylating the ubiquitin E3 ligase beta-TrCP. Nat Microbiol. 2019:4(11):1872-84.

27. Aravind L. The WWE domain: a common interaction module in protein ubiquitination and ADP ribosylation. Trends Biochem Sci. 2001;26(5):273-5

28. Gibson BA, Kraus WL. New insights into the molecular and cellular functions of poly(ADP-ribose) and PARPs. Nat Rev Mol Cell Biol. 2012;13(7):411-24.

29. He F, Tsuda K, Takahashi M, Kuwasako K, Terada T, Shirouzu M, et al. Structural insight into the interaction of ADP-ribose with the PARP WWE domains. FEBS lett. 2012;586(21):3858-64.

30. Guo X, Ma J, Sun J, Gao G. The zinc-finger antiviral protein recruits the RNA processing exosome to degrade the target mRNA. Proc Natl Acad Sci USA. 2007;104(1):151-6.

31. Meyer-Ficca ML, Ihara M, Bader JJ, Leu NA, Beneke S, Meyer RG. Spermatid head elongation with normal nuclear shaping requires ADP-ribosyltransferase PARP11 (ARTD11) in mice. Biol Reprod. 2015;92(3):80.

32. Kirby IT, Kojic A, Arnold MR, Thorsell AG, Karlberg T, Vermehren-Schmae$\operatorname{dick} A$, et al. A potent and selective PARP11 inhibitor suggests coupling between cellular localization and catalytic activity. Cell Chem Biol. 2018:25(12):1547-53 e12.

33. Zhang FC, Li XF, Deng YQ, Tong YG, Qin CF. Excretion of infectious Zika virus in urine. Lancet Infect Dis. 2016;16(6):641-2.

34. Liu SY, Aliyari R, Chikere K, Li G, Marsden MD, Smith JK, et al. Interferoninducible cholesterol-25-hydroxylase broadly inhibits viral entry by production of 25-hydroxycholesterol. Immunity. 2013;38(1):92-105.

35. Cong L, Ran FA, Cox D, Lin S, Barretto R, Habib N, et al. Multiplex genome engineering using CRISPR/Cas systems. Science. 2013;339(6121):819-23.

36. Mali P, Yang L, Esvelt KM, Aach J, Guell M, DiCarlo JE, et al. RNA-guided human genome engineering via Cas9. Science. 2013:339(6121):823-6.

37. Johnson BW, Russell BJ, Lanciotti RS. Serotype-specific detection of dengue viruses in a fourplex real-time reverse transcriptase PCR assay. J Clin Microbiol. 2005;43(10):4977-83.

38. Deng YQ, Zhao H, Li XF, Zhang NN, Liu ZY, Jiang T, et al. Isolation, identification and genomic characterization of the Asian lineage Zika virus imported to China. Sci China Life Sci. 2016;59(4):428-30.

\section{Publisher's Note}

Springer Nature remains neutral with regard to jurisdictional claims in published maps and institutional affiliations.

Ready to submit your research? Choose BMC and benefit from:

- fast, convenient online submission

- thorough peer review by experienced researchers in your field

- rapid publication on acceptance

- support for research data, including large and complex data types

- gold Open Access which fosters wider collaboration and increased citations

- maximum visibility for your research: over 100M website views per year

At $\mathrm{BMC}$, research is always in progress.

Learn more biomedcentral.com/submissions 